\title{
Dynamic Output Feedback Controller Synthesis using an LMI-based Strictly Negative Imaginary Framework
}

DOI:

10.1109/MED.2019.8798493

\section{Document Version}

Accepted author manuscript

Link to publication record in Manchester Research Explorer

\section{Citation for published version (APA):}

Kurawa, S., Bhowmick, P., \& Lanzon, A. (2019). Dynamic Output Feedback Controller Synthesis using an LMIbased Strictly Negative Imaginary Framework. In Proceedings of the 27th Mediterranean Conference on Control and Automation https://doi.org/10.1109/MED.2019.8798493

\section{Published in:}

Proceedings of the 27th Mediterranean Conference on Control and Automation

\section{Citing this paper}

Please note that where the full-text provided on Manchester Research Explorer is the Author Accepted Manuscript or Proof version this may differ from the final Published version. If citing, it is advised that you check and use the publisher's definitive version.

\section{General rights}

Copyright and moral rights for the publications made accessible in the Research Explorer are retained by the authors and/or other copyright owners and it is a condition of accessing publications that users recognise and abide by the legal requirements associated with these rights.

\section{Takedown policy}

If you believe that this document breaches copyright please refer to the University of Manchester's Takedown Procedures [http://man.ac.uk/04Y6Bo] or contact uml.scholarlycommunications@manchester.ac.uk providing relevant details, so we can investigate your claim.

\section{OPEN ACCESS}




\title{
Dynamic Output Feedback Controller Synthesis using an LMI-based $\alpha-$ Strictly Negative Imaginary Framework
}

\author{
Suleiman Kurawa, Parijat Bhowmick and Alexander Lanzon
}

\begin{abstract}
This paper deals with the dynamic output feedback controller synthesis utilizing the $\alpha$ - strictly negative imaginary systems property. The proposed scheme ensures robust stability in closed-loop against the set of all stable, strictly proper negative imaginary uncertainties that satisfies the DC loop gain condition pertaining to negative imaginary stability. In addition to that, a prescribed decay rate in the closedloop time response is also enforced via $\alpha$ - pole placement. Two illustrative examples have been studied to demonstrate the usefulness of the proposed controller synthesis scheme.
\end{abstract}

\section{INTRODUCTION}

The theory of negative imaginary (NI) systems was motivated by the study of inertial systems with colocated force actuator and position sensor [1], [3]. In a single-input-singleoutput (SISO) setting, a stable transfer function is said to have the NI (resp., strictly NI or SNI) frequency response if its Nyquist plot lies below (resp., strictly below) the real axis of the complex plane in the open frequency interval from zero to infinity [1]. In contrast to positive real (PR) systems, NI systems can have relative degree of up to two and they can also have non-minimum phase zeros [8]. Due to virtue of the simple internal stability condition for interconnected NI and SNI systems that depends primarily on the DC loop gain information [1], the NI system theory finds immense applications in controller design especially for mechanical and mechatronic systems. For example, it has been applied in vibration control of a flexible robotic arm [10], in motion control of a robotic arm with unknown parameters [6], in vehicle platooning [4], in control of a DC servo motor [7], in nano-positioning of an atomic force microscope [11], and in position control of a swing-arm hard disk drive [15].

So far in the literature, a handful of effort has been dedicated towards the problem of NI controller synthesis. For example, an LMI-based state feedback synthesis imposing closed-loop NI property is explored in [3], [6]. While in [11], the state feedback synthesis has been done to enforce closedloop NI/SNI property using an algebraic Riccati equation (ARE) based approach. In [13], the authors introduce a static state feedback and a dynamic output feedback controller synthesis schemes imposing closed-loop NI property. In [15],

This work was supported by the Engineering and Physical Sciences Research Council (EPSRC) [grant number EP/R008876/1] and the Petroleum Technology Development Fund overseas scholarship sheme. All research data supporting this publication are directly available within this publication.

Suleiman Kurawa, Parijat Bhowmick and Alexander Lanzon are with the Control Systems Centre, School of Electrical and Electronic Engineering, University of Manchester, Sackville Street, Manchester M13 9PL, U.K. Suleiman.Kurawa@postgrad.manchester.ac.uk,

Parijat.Bhowmick@manchester.ac.uk,

Alexander. Lanzon@manchester.ac.uk. a dynamic output feedback control framework is proposed for systems with PR uncertainty which is then applied to systems with stable NI uncertainty by transforming the NI uncertainty into PR framework. Very recently, in [16] and [17], strictly negative imaginary controller synthesis problems considering both state and output feedback have been addressed applying an ARE-based approach.

Pertaining to NI synthesis that facilitates some transient performance (e.g. decay rate) via closed-loop poleplacement, the work of [12] can be referred which provides a state feedback NI controller synthesis technique exploiting the notion of $\alpha$ - and $\mathscr{D}$ - pole placement introduced in [19]. However, in many practical applications, some of the states may not be accessible for external measurement and hence, output feedback sometimes outperforms state feedback. Nevertheless, regional pole placement, or pole placement in general, provides a way of achieving time domain performance such as reducing peak overshoot and settling time, increasing decay rate, etc. [20].

Being motivated by the aforementioned developments, this paper proposes an LMI-based procedure to design a dynamic output feedback controller applying the $\alpha$ - SNI framework that ensures robust stability against all stable, strictly proper, NI uncertainties. Furthermore, it is shown via examples that the designed controller can also satisfy a certain level of robust $\mathscr{H}_{\infty}$ performance applying $\mu$-analysis.

\section{PRELIMINARIES}

This section recalls some definitions, lemmas and technical results from the literature important for developing the main results. The first definition for NI systems was given in [1] for stable systems. This was then extended in [2] to marginally-stable systems having poles in the closed left-half plane excluding the origin. In this paper, we have used the definition of NI systems according to [2].

Definition 1: (NI System) [2] A square, real, rational and proper transfer function matrix $M(s)$ is said to be NI if

1) $M(s)$ has no poles in $\Re\{s\}>0$;

2) $j\left[M(j \omega)-M(j \omega)^{*}\right] \geq 0$ for all $\omega \in(0, \infty)$ such that $j \omega$ is not a pole of $M(s)$;

3) If $j \omega_{0}$ with $\omega_{0} \in(0, \infty)$ is a pole of $M(s)$, it is at most a simple pole and the residue matrix $K_{0}=\lim _{s \rightarrow j \omega_{0}} j(s-$ $\left.j \omega_{0}\right) M(s)$ is Hermitian and positive semidefinite.

The definition of NI systems have been further extended in [8], [10] to account for NI systems with single and double poles at the origin. See also [14] for some recent feedback stability analysis results allowing poles at the origin. Let us recall the definition of SNI systems. 
Definition 2: (SNI System) [1] Let $M(s)$ be a square, real, rational and proper transfer function matrix. $M(s)$ is said to be SNI if $M(s)$ has no poles in $\mathfrak{R}[s] \geq 0$ and $j[M(j \omega)-$ $\left.M(j \omega)^{*}\right]>0$ for all $\omega \in(0, \infty)$.

The following lemma characterizes the strongly strict negative imaginary (SSNI) systems introduced in [5].

Lemma 1: [5] Let $\left[\begin{array}{l|l}A & B \\ \hline C & D\end{array}\right]$ be a state-space realization of a real, rational, proper and square transfer function matrix $M(s)$. Assume, $M(s)-M(-s)^{T}$ has full normal rank and the pair $(A, C)$ is observable. Then, $A$ is Hurwitz and $M(s)$ is SNI with $\lim _{\omega \rightarrow 0} j \frac{1}{\omega}\left[M(j \omega)-M(j \omega)^{*}\right]>0$ and $\lim _{\omega \rightarrow \infty} j \omega\left[M(j \omega)-M(j \omega)^{*}\right]>0$ if and only if $D=D^{T}$ and there exists $Y=Y^{T}>0$ such that

$$
A Y+Y A^{T}<0 \text { and } B+A Y C^{T}=0
$$

The conditions necessary and sufficient for the internal stability of a positive feedback interconnection of stable NI and SNI systems are next presented.

Theorem 1: [1] Let $M(s)$ be SNI and $\gamma>0$. Then, the positive feedback interconnection of $M(s)$ and $\Delta(s)$ is internally stable for all stable NI systems $\Delta(s)$ that satisfy $\Delta(\infty) \geq 0, \Delta(\infty) M(\infty)=0$ and $\lambda_{\max }[\Delta(0)]<\gamma^{-1}$ if and only if $\lambda_{\max }[M(0)] \leq \gamma$.

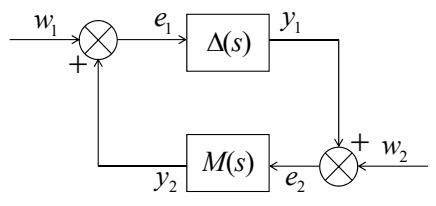

(a)

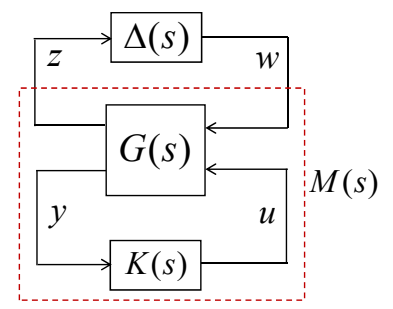

(b)
Fig. 1: (a) Positive feedback interconnection of two NI systems; (b) $M-\Delta$ configuration for robust stability analysis.

Towards this end, we set the notations for $\alpha$ - strictly negative imaginary (abbreviated as $\alpha-\mathrm{SNI}$ ) systems. The $\alpha-$ SNI subclass is closely related to the SSNI class of systems [5] and is defined by a state-space characterization.

Definition 3: Let $D=D^{T}$ and $\alpha>0$. Then, $R(s)=$ $\left[\begin{array}{c|c}A & B \\ \hline C & D\end{array}\right]$ is said to be $\alpha-$ SNI if there exists a real matrix $Y=Y^{T}>0$ such that

$$
A Y+Y A^{T}+2 \alpha Y \leq 0 \quad \text { and } B+A Y C^{T}=0 .
$$

We now provide some remarks which explore the properties of $\alpha-$ SNI systems and find the connections between $\alpha-$ SNI and SSNI systems properties.

Remark 1: The $\alpha-$ SNI systems are inherently stable. This can be readily established from (2), which implies $A Y+Y A^{T}<0$ for $\alpha>0$ and $Y>0$, which in turn ensures Hurwitzness of $A$ applying [18, Lemma 3.19].

Note that the definition of $\alpha-$ SNI systems does not require a minimal state-space realisation of the underlying system. In this context, the literature [6], [16] may be referred where it is shown that most of the analysis and synthesis results associated with NI, SNI and SSNI systems theory remain applicable in case of non-minimal system realization.

Remark 2: From Definition 3, one may think that the set of the $\alpha-$ SNI systems is a subset of the SSNI class having poles in $\mathfrak{R}[s] \leq-\alpha$. But, unlike SSNI systems [5], $\alpha-\mathrm{SNI}$ system property does not impose any restrictions on the statespace realisation. It can be concluded that the set of $\alpha-$ SNI systems, say $R(s)$, with a completely observable statespace realisation and $R(s)-R(-s)^{T}$ having full normal rank belongs to the SSNI class.

Note that in case of $\alpha$ - SNI systems the full normal rank constraint on $R(s)-R(-s)^{T}$ is implied by (2) when the $B$ matrix has full column rank. It is proved in the following lemma. The same conclusion applies to Lemma 1 as well.

Lemma 2: Let $R(s)=\left[\begin{array}{c|c}A & B \\ \hline C & D\end{array}\right]$ be an $(m \times m) \alpha-\mathrm{SNI}$ system with $\operatorname{rank}[B]=m$. Then, $R(s)-R(-s)^{T}$ has full normal rank.

Proof. For a given $\alpha>0$ and $Y>0$, (2) implies $A Y+$ $Y A^{T}<0$. Then there exists a square and non-singular matrix $L$ such that $A Y+Y A^{T}=-L^{T} L$. For these $L$ and $Y$, the transfer function matrix $N(s)=\left[\begin{array}{c|c}A & B \\ \hline L Y^{-1} A^{-1} & 0\end{array}\right]$ acquires full column rank at $s=j \omega$ for all $\omega \in \mathbb{R}$ since $A$ is Hurwitz and $\operatorname{rank}[B]=m$ via assumption and $\operatorname{rank}\left[L Y^{-1} A^{-1}\right]=n$. It implies from [2, Corollary 1]

$$
j \omega\left[R(j \omega)-R(j \omega)^{*}\right]=\omega^{2} N(j \omega)^{*} N(j \omega)>0
$$

for all $\omega \in \mathbb{R} \backslash\{0\}$ and $R(0)-R(0)^{T}=0$ since $R(0)=$ $C Y C^{T}+D=R(0)^{T}$. This implies that there does not exist any continuum interval of $\omega \in \mathbb{R}$ for which $\operatorname{det}\left[R(j \omega)-R(j \omega)^{*}\right]$ remains zero. This in turn ensures that $R(s)-R(-s)^{T}$ must have full normal rank. Note minimality is not required.

In the next section, we will introduce a dynamic output feedback controller synthesis framework exploiting the $\alpha-$ SNI property.

\section{MAIN RESULTS}

\section{A. Problem Formulation}

Consider an LTI generalized plant $G$ described by the following state-space equations

$$
G:\left\{\begin{array}{l}
\dot{x}=A x+B_{1} w+B_{2} u, \\
z=C_{1} x+D_{11} w+D_{12} u, \\
y=C_{2} x+D_{21} w,
\end{array}\right.
$$

where $x(t) \in \mathbb{R}^{n}$ is the state vector of the generalized plant, $u(t) \in \mathbb{R}^{n_{u}}$ represents the control input, $y(t) \in \mathbb{R}^{n_{y}}$ is the measured output, $w(t) \in \mathbb{R}^{m}$ is the exogenous input and $z(t) \in$ $\mathbb{R}^{m}$ is the objective signal. The matrices $A \in \mathbb{R}^{n \times n}, B_{1} \in$ $\mathbb{R}^{n \times m}, B_{2} \in \mathbb{R}^{n \times n_{u}}, C_{1} \in \mathbb{R}^{m \times n}, C_{2} \in \mathbb{R}^{n_{y} \times n}, D_{11}$ and $D_{21}$ are all constant and known. Assume that $D_{12}=0,\left(A, B_{2}\right)$ is stabilizable and $\left(A, C_{2}\right)$ is detectable. The aim is to synthesize a full-order dynamic output feedback controller

$$
K:\left\{\begin{aligned}
\dot{x}_{c} & =A_{c} x_{c}+B_{c} y \\
u & =C_{c} x_{c}+D_{c} y
\end{aligned}\right.
$$


such that the nominal closed-loop system $M(s)$ is $\alpha-\mathrm{SNI}$ and the generalized plant $G$ is robustly stabilized against all stable, strictly proper, NI uncertainties $\Delta(s)$ with $\Delta(0) \leq \gamma^{-1}$ for a given $\gamma>0$. The state-space realization of the controller synthesized nominal closed-loop system $M(s)$, as shown in Fig. 1b, from $w$ to $z$ is given by

$M(s)=\left[\begin{array}{c|c}A_{c l} & B_{c l} \\ \hline C_{c l} & D_{c l}\end{array}\right]=\left[\begin{array}{cc|c}A+B_{2} D_{c} C_{2} & B_{2} C_{c} & B_{1}+B_{2} D_{c} D_{21} \\ B_{c} C_{2} & A_{c} & B_{c} D_{21} \\ \hline C_{1} & 0 & D_{11}\end{array}\right]$

\section{B. Controller synthesis using $\alpha-$ SNI framework}

This subsection provides the main contribution of this paper. Theorem 2 gives a set of sufficient conditions required for the existence of a dynamic output feedback controller $K(s)$ which makes the nominal closed-loop system given in (6) $\alpha-$ SNI with a given $\alpha>0$ and maintains closedloop stability in presence of any stable, strictly proper, NI uncertainty satisfying the DC gain condition.

Theorem 2: Let a generalized plant $G$ be given by (4) with $D_{11}=D_{11}^{T}, D_{12}=0,\left(A, B_{2}\right)$ stabilizable and $\left(A, C_{2}\right)$ detectable. Let $\gamma>0, \alpha>0$ and $m \leq 2 n$. Suppose there exist matrices $\hat{A} \in \mathbb{R}^{n \times n}, \hat{B} \in \mathbb{R}^{n \times n_{y}}, \hat{C} \in \mathbb{R}^{n_{u} \times n}, \hat{D} \in \mathbb{R}^{n_{u} \times n_{y}}$ and symmetric matrices $P \in \mathbb{R}^{n \times n}$ and $X \in \mathbb{R}^{n \times n}$ such that

$$
\begin{aligned}
& {\left[\begin{array}{cc:c}
\Phi_{11}+2 \alpha P & \Phi_{12}+2 \alpha I & P B_{1}+\hat{B} D_{21}+\hat{A} C_{1}^{T} \\
\hdashline & \Phi_{22}+2 \alpha X & \Phi_{23} \\
\hdashline \star & x^{\star} & 0
\end{array}\right] \leq 0} \\
& {\left[\begin{array}{cc}
P & I \\
I & X
\end{array}\right]>0} \\
& \text { and } C_{1} X C_{1}^{T}+D_{11}<\gamma I
\end{aligned}
$$

with the following shorthand

$$
\begin{aligned}
& \Phi_{11}=P A+P A^{T}+\hat{B} C_{2}+C_{2}^{T} \hat{B}^{T}, \\
& \Phi_{12}=\hat{A}+\left(A+B_{2} \hat{D} C_{2}\right)^{T}, \\
& \Phi_{22}=X A^{T}+A X+B_{2} \hat{C}+\hat{C}^{T} B_{2}^{T}, \\
& \Phi_{23}=B_{1}+B_{2} \hat{D} D_{21}+A X C_{1}^{T}+B_{2} \hat{C} C_{1}^{T},
\end{aligned}
$$

and the symbol $\star$ denotes the elements due to symmetry. Then, an internally stabilizing controller $K(s)$ is given by (5) where

$$
\begin{aligned}
D_{c}= & \hat{D}, \\
C_{c}= & \left(\hat{C}-D_{c} C_{2} X\right) M^{-T}, \\
B_{c}= & N^{-1}\left(\hat{B}-P B_{2} D_{c}\right), \\
A_{c}= & N^{-1}\left(\hat{A}-P A X-N B_{c} C_{2} X\right. \\
& \left.\quad-P B_{2} C_{c} M^{T}-P B_{2} D_{c} C_{2} X\right) M^{-T},
\end{aligned}
$$

and $M$ and $N$ are square and non-singular solutions of the algebraic equation $M N^{T}=I-X P$. This controller $K(s)$ forms a closed-loop system $M(s)$, expressed as in (6), which is $\alpha-$ SNI and is robust to all stable, strictly proper, NI uncertainty $\Delta(s)$ satisfying $\lambda_{\max }[\Delta(0)] \leq \gamma^{-1}$.

Proof. First note that $D_{c l}=D_{c l}^{T}$ in (6) and $m \leq 2 n$. The proof will proceed via the following steps which establishes that for $M(s)=\left(\begin{array}{c|c}A_{c l} & B_{c l} \\ \hline C_{c l} & D_{c l}\end{array}\right)$ to be $\alpha-$ SNI with a given $\alpha>0$, conditions (7)-(9) need to be satisfied.

Step 1: From Definition 3, $M(s)=\left(\begin{array}{l|l}A_{c l} & B_{c l} \\ \hline C_{c l} & D_{c l}\end{array}\right)$ is $\alpha-$ SNI with a given $\alpha>0$ if there exists $Y=Y^{T}>0$ such that

$$
\left(\begin{array}{cc}
A_{c l} Y+Y A_{c l}^{T}+2 \alpha Y & \star \\
B_{c l}^{T}+C_{c l} Y A_{c l}^{T} & 0
\end{array}\right) \leq 0 .
$$

Step 2: Since inequality (12) is not in LMI form due to presence of the terms containing products of unknown controller variables, a linearising change in controller variables [19], [20] is required to transform (12) into LMI form. Partition the closed-loop Lyapunov matrix $Y$ and $Y^{-1}$ as follows:

$$
Y=\left(\begin{array}{cc}
X & M \\
M^{T} & \bullet
\end{array}\right) \quad \text { and } \quad Y^{-1}=\left(\begin{array}{cc}
P & N \\
N^{T} & \bullet
\end{array}\right),
$$

where $X$ and $P$ are symmetric $n \times n$ matrices and the symbol - represents matrices that are not explicitly used in the linearization process. Note $Y^{-1}$ exists since $Y>0$ via (8) which has been explained subsequently in step 4. Note also that $X, P, M, N$ are not independent variables but must satisfy $X P+M N^{T}=I$ (see [19], [20] for details). Since, $M$ and $N$ are square and non-singular, the following block matrices

$$
\Pi_{1}=\left(\begin{array}{cc}
P & I \\
N^{T} & 0
\end{array}\right) \text { and } \Pi_{2}=\left(\begin{array}{cc}
I & X \\
0 & M^{T}
\end{array}\right)
$$

are non-singular. $\Pi_{1}$ and $\Pi_{2}$ are related through the expression

$$
Y \Pi_{1}=\Pi_{2}
$$

which is obtained from $Y Y^{-1}=I$. The change of controller variables are defined as

$$
\left\{\begin{array}{l}
\hat{A}=P A X+N B_{c} C_{2} X+P B_{2} C_{c} M^{T}+P B_{2} D_{c} C_{2} X+N A_{c} M^{T}, \\
\hat{B}=N B_{c}+P B_{2} D_{c}, \\
\hat{C}=D_{c} C_{2} X+C_{c} M^{T}, \quad \text { and } \\
\hat{D}=D_{c} .
\end{array}\right.
$$

Step 3: Applying a congruence transformation on (12) with the block diagonal matrix $\operatorname{diag}\left\{\Pi_{1}, I\right\}$ and using (15), we obtain

$$
\left[\begin{array}{cc}
\Pi_{1}^{T} A_{c l} \Pi_{2}+\Pi_{2}^{T} A_{c l}^{T} \Pi_{1}+2 \alpha \Pi_{1}^{T} \Pi_{2} & \star \\
\left(B_{c l}^{T}+C_{c l} Y A_{c l}^{T}\right) \Pi_{1} & 0
\end{array}\right] \leq 0 .
$$

Simplifying all the product terms and substituting into (17) the linearizing change of controller variables given in (16), we get back condition (7), that is,

$$
\left[\begin{array}{cc:c}
\Phi_{11}+2 \alpha P & \Phi_{12}+2 \alpha I & P B_{1}+\hat{B} D_{21}+\hat{A} C_{1}^{T} \\
\hdashline \star & \Phi_{22}+2 \alpha X & \Phi_{23} \ldots \\
\hdashline \star & \vdots & 0
\end{array}\right] \leq 0 .
$$

Step 4: Positive definiteness of the closed-loop Lyapunov matrix $Y=\left(\begin{array}{cc}X & M \\ M^{T} & \bullet\end{array}\right)$ is guaranteed by (8) via the congruence transformation shown below

$$
\Pi_{1}^{T} Y \Pi_{1}=\left(\begin{array}{cc}
P & I \\
I & X
\end{array}\right)>0
$$


Step 5: The inequality condition (9) is equivalent to $M(0)<\gamma I$ since $M(0)=C_{c l} Y C_{c l}^{T}+D_{11}$. This in turn implies $\lambda_{\max }[M(0) \Delta(0)]<1$ via [1]. Thus, the interconnection of $M(s)$ being $\alpha-$ SNI and $\Delta(s)$ being stable NI satisfies all the assumptions of Theorem 1 as well as the DC loop gain condition. Therefore, the interconnection is robustly stable. This completes the proof.

Remark 3: In order to find square and non-singular solutions of $M$ and $N$ from the expression $M N^{T}=I-X P$, methods such as $Q-R$ factorisation, Cholesky factorisation or Eigen-decomposition can be used. But the easiest solution is to choose $M=I$ and accordingly, $N=I-P X$. This choice of $M$ and $N$ rules out the possibility of getting ill-conditioned solutions in Matlab due to computational issues.

\section{ILLUSTRATIVE EXAMPLES}

In this section, we will present two illustrative examples to elucidate the usefulness of the proposed synthesis technique.

\section{A. Example 1}

We reconsider the model of an uncertain flexible structure system with colocated position sensor and force actuator, as shown in Fig. 2, originally studied in [3], [6] followed later by [13]. The generalized plant model $G$ of the physical

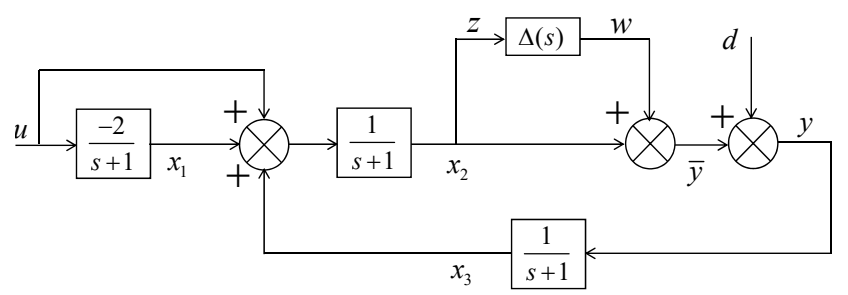

Fig. 2: Block diagram of the simplified model of an uncertain flexible structure system taken in [6].

system is governed by the following state-space equations. The output of the system $y(t)$ is subjected to some bounded disturbance $d(t) \in \mathbb{R}$.

$$
\begin{gathered}
G:\left\{\left[\begin{array}{c}
\dot{x}_{1} \\
\dot{x}_{2} \\
\dot{x}_{3}
\end{array}\right]=\left[\begin{array}{ccc}
-1 & 0 & 0 \\
1 & -1 & 1 \\
0 & 1 & -1
\end{array}\right]\left[\begin{array}{l}
x_{1} \\
x_{2} \\
x_{3}
\end{array}\right]+\right. \\
{\left[\begin{array}{cc}
0 & 0 \\
0 & 0 \\
1 & 1
\end{array}\right]\left[\begin{array}{l}
w \\
d
\end{array}\right]+\left[\begin{array}{c}
-2 \\
1 \\
0
\end{array}\right] u,} \\
{\left[\begin{array}{c}
z \\
y
\end{array}\right]=\left[\begin{array}{lll}
0 & 1 & 0 \\
0 & 1 & 0
\end{array}\right]\left[\begin{array}{l}
x_{1} \\
x_{2} \\
x_{3}
\end{array}\right]+\left[\begin{array}{ll}
0 & 0 \\
1 & 1
\end{array}\right]\left[\begin{array}{l}
w \\
d
\end{array}\right],}
\end{gathered}
$$

and $W(s)=\Delta(s) Z(s)$, where $W(s)$ and $Z(s)$ are the Laplace transform of $w(t)$ and $z(t)$ respectively. In line with [6], $\Delta(s)$ is an SNI uncertainty with $\Delta(\infty)=0$ and $\Delta(0) \leq 1$.

Part I. The control objective is to synthesize a dynamic output feedback controller $K(s)$ such that the nominal closedloop system $M(s)$ becomes $\alpha-$ SNI and the generalized plant $G$ remains stable closed-loop in presence of any $\Delta(s)$ defined above. Choosing $\alpha=0.8$ and applying Theorem 2, we obtain a feasible solution set of matrices

$$
\begin{aligned}
& P=\left[\begin{array}{ccc}
126.3650 & 15.0662 & 83.8253 \\
15.0662 & 7.8543 & 15.0240 \\
83.8253 & 15.0240 & 126.6103
\end{array}\right]>0, \\
& X=\left[\begin{array}{ccc}
33.3391 & -2.0000 & -31.8531 \\
-2.0000 & 0.5764 & 1.5764 \\
-31.8531 & 1.5764 & 30.8991
\end{array}\right]>0,
\end{aligned}
$$

and $\hat{A}, \hat{B}, \hat{C}, \hat{D}$ using the CVX toolbox [21]. We fix $M=I$ and hence, $N=I-P X$. The controller matrices $A_{c}, B_{c}, C_{c}$ and $D_{c}$ are then uniquely reconstructed for this $M$ and $N$ using the relations (11a)-(11d). The controller $K(s)$ is computed as

$$
K(s)=\frac{3.7491(s+1)(s+1.345)(s+6.358)}{(s+36.45)(s+1.386)(s+1.001)}
$$

which constitutes the nominal closed-loop system

$$
M(s)=\frac{3.749 s^{5}+33.63 s^{4}+}{97.04 s^{3}+126.6 s^{2}+77.97 s+18.48}
$$

from $w$ to $z$. The closed-loop poles are given by $\lambda_{i}\left(A_{c l}\right)=$ $\{-33.1278,-1.1847,-0.9259 \pm j 0.1564,-0.9264,-1.00\}$. It can be readily verified that $M(s)$ is an $\alpha-$ SNI transfer function with $\mathfrak{R}\left[\lambda_{i}\left[A_{c l}\right]\right]<-0.8$ for all $i$. The Nyquist plot of $M(s)$ given in Fig. 3a also reflects that $M(s)$ is an SNI transfer function. Now, we find $M(0)=0.5764$ and hence, $M(0) \Delta(0)=0.5764<1$, which ensures robust stability in closed-loop against the given set of $\Delta(s)$ having $\Delta(0) \leq$ 1 via Theorem 2. In order to show the applicability of

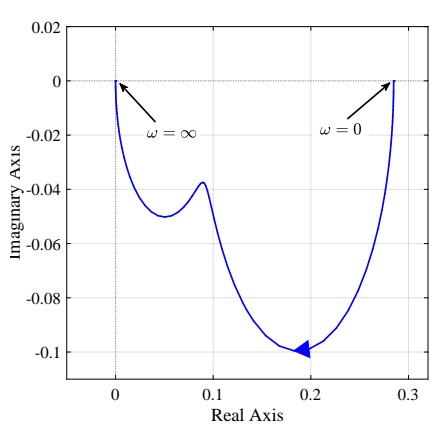

(a)

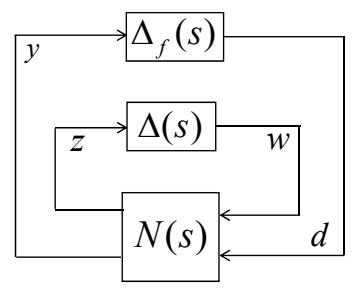

(b)
Fig. 3: (a) Nyquist plot of the $\alpha-$ SNI transfer function $M(s)$ obtained in Example 1; (b) LFT configuration for robust performance problem by augmenting a fictitious uncertainty $\Delta_{f}(s)$ with the stable NI uncertainty $\Delta(s)$.

the proposed synthesis scheme, we study the disturbanceattenuation problem in presence of two arbitrarily chosen strictly proper SNI uncertainties given by $\Delta_{1}(s)=\frac{1}{s+2}$ and $\Delta_{2}=\frac{1}{s+20}$. A pulse disturbance having amplitude 0.1 and $T_{o n}=1 \mathrm{~s}$ is applied to the system under all zero initial condition. Figures $4 \mathrm{a}-4 \mathrm{~d}$ compare the closed-loop time response corresponding to the nominal case and in presence of the 
uncertainties. It is observed that the designed controller $K(s)$ ensures closed-loop stability and also provides satisfactory transient performance in presence of the uncertainty. The figures reveal that the percentage deviation from nominal to perturbed condition in each of the states as well as the output $y(t)$ remains within $5 \%$ only. Moreover, despite the presence of uncertainty, the control effort $u(t)$ increases to a negligible extent with respect to nominal level. The plot of $u(t)$ has not been included due to space constraint.

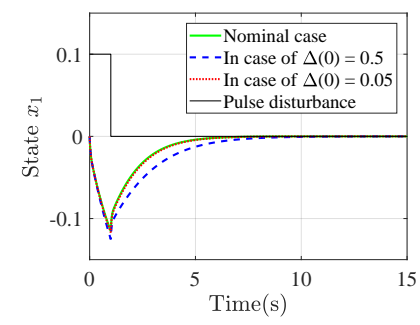

(a)

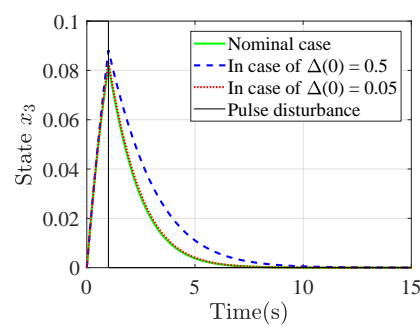

(c)

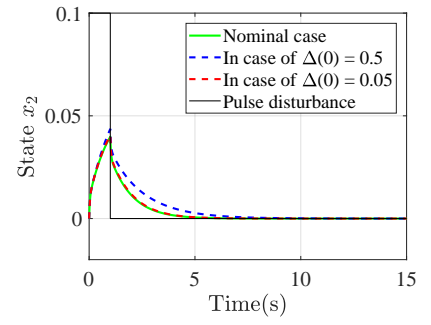

(b)

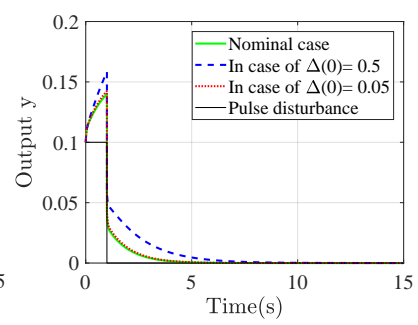

(d)
Fig. 4: Closed-loop time responses of the designed system under the nominal as well as perturbed condition subjected to a pulse disturbance and zero initial condition: (a) State $x_{1}(t)$, (b) State $x_{2}(t)$, (c) State $x_{3}(t)$, (d) System output $y(t)$.

Part II. Apart from the time domain performance analysis, we would also like to quantify and measure the robust $\mathscr{H}_{\infty}$ performance of the closed-loop system output (y) from the disturbance $(d)$ achieved by the designed controller $K(s)$. Theoretically, robust $\mathscr{H}_{\infty}$ performance (RP) is analysed [18] by recasting the robust performance problem into a robust stability problem with respect to the augmented, twoblock uncertainty $\Delta_{p}(s)=\left[\begin{array}{cc}\Delta(s) & 0 \\ 0 & \Delta_{f}(s)\end{array}\right]$, where $\Delta_{f}(s) \in$ $\mathscr{R} \mathscr{H}_{\infty}^{n_{d} \times n_{y}}$ is a fictitious uncertainty and $\Delta(s) \in \mathscr{R} \mathscr{H}_{\infty}^{m \times m}$ is the physical uncertainty as depicted in Fig. 3b. To measure the robust $\mathscr{H}_{\infty}$ performance, $\mu$-analysis technique is invoked. According to [18, Theorem 11.9], robust $\mathscr{H}_{\infty}$ performance with level $\beta$ of the closed-loop system shown in Fig. $3 \mathrm{~b}$ is guaranteed for all stable $\Delta(s)$ with $\|\Delta(s)\|_{\infty}<\frac{1}{\beta}$ for a given $\beta>0$ if and only if

$$
\sup _{\omega \in \mathbb{R}} \mu_{\Delta_{p}}(N(j \omega)) \leq \beta,
$$

where $N(s)$ represents the transfer function mapping from $\left[\begin{array}{l}w \\ d\end{array}\right]$ to $\left[\begin{array}{l}z \\ y\end{array}\right]$. Following this approach, in the present example, we compute an upper bound to $\mu_{\Delta_{p}}(N(j \omega))$ using the Matlab Robust Control Toolbox for the frequency interval $\omega \in\left[10^{-4}, 10^{4}\right]$ and find $\sup _{\Delta_{P}}(N(j \omega)) \leq 2.1529$. $\omega \in\left[10^{-4}, 10^{4}\right]$

We also calculate $\left\|N_{11}(s)\right\|_{\infty}=\|M(s)\|_{\infty}=0.5764$. It signifies that, to satisfy robust stability alone (via Small-gain Theorem [18]), $\|\Delta(s)\|_{\infty}<\frac{1}{0.5764}=1.7349$; while, to ensure the robust $\mathscr{H}_{\infty}$ performance with level 2.1529 via $\mu$-analysis, $\|\Delta(s)\|_{\infty}<\frac{1}{2.1529}=0.4645$ is required. On the contrary, to ensure robust stability according to NI-SNI stability condition, $\Delta(s)$ needs to satisfy $\Delta(0)<\frac{1}{M(0)}=1.7349$ as $M(0)=0.5746$ (as found in Part I). Now, $\|\Delta(s)\|_{\infty}<0.4645$ is equivalent to $|\Delta(j \omega)|<0.4645 \forall \omega \in \mathbb{R}$ which implies that the latter region is a significant restriction of the former region. Thus the $\alpha-$ SNI synthesis allows a bigger set of uncertainties compared to the set satisfying the robust $\mathscr{H}_{\infty}$ performance.

\section{B. Example 2}

Here, we adopt the following MIMO example from [13] being inspired by [9], where the same example was studied to show an application of a decentralized static output feedback controller. The generalized plant $G$ is expressed via (4) with the following state-space matrices

$A=\left[\begin{array}{ccccc}-4 & 0 & -2 & 0 & 0 \\ 0 & -2 & 0 & 2 & 0 \\ 0 & 0 & -2 & 0 & -1 \\ 0 & -2 & 0 & -1 & 0 \\ 3 & 0 & -2 & 0 & -1\end{array}\right], B_{1}=\left[\begin{array}{l}1 \\ 1 \\ 1 \\ 1 \\ 1\end{array}\right]$,
$C_{1}^{T}=\left[\begin{array}{l}0 \\ 1 \\ 0 \\ 0 \\ 0\end{array}\right], B_{2}=\left[\begin{array}{ccc}1 & 0 & 0 \\ 1 & 0 & 0 \\ 0 & 0 & 0 \\ 0 & 1 & 0 \\ 0 & 0 & 1\end{array}\right], C_{2}=\left[\begin{array}{lllll}1 & 0 & 0 & 0 & 0 \\ 0 & 1 & 0 & 0 & 0 \\ 0 & 0 & 0 & 0 & 1\end{array}\right]$ and $D_{11}=0, D_{12}=0_{1 \times 4}, D_{21}=0_{3 \times 1}$. The uncertainty $\Delta(s)$ is assumed to be any stable, strictly proper, NI transfer function with $\Delta(0) \leq 3.6$. Similar to Example 1, the control objective is to synthesize an output feedback controller $K(s)$ which internally stabilizes $G$ and renders $M(s)$ from $w$ to $z \alpha-$ SNI. Choosing $\alpha=2$, we apply Theorem 2 on $G$ and obtain a feasible solution set of matrices

$\begin{aligned} X & =\left[\begin{array}{ccccc}24.58 & -0.06 & -9.69 & -2.83 & -7.73 \\ -0.06 & 0.25 & 0.34 & 0.03 & 0.32 \\ -9.69 & 0.34 & 24.50 & -5.65 & 10.07 \\ -2.83 & 0.03 & -5.65 & 31.85 & -1.45 \\ -7.73 & 0.32 & 10.07 & -1.45 & 34.22\end{array}\right]>0 \\ P & =\left[\begin{array}{ccccc}21.12 & -7.37 & 1.86 & -4.69 & -7.69 \\ -7.37 & 53.42 & -10.22 & -23.68 & -7.41 \\ 1.86 & -10.22 & 11.45 & -2.81 & 1.85 \\ -4.69 & -23.68 & -2.81 & 39.11 & -4.68 \\ -7.69 & -7.41 & 1.85 & -4.68 & 21.14\end{array}\right]>0\end{aligned}$

and $\hat{A}, \hat{B}, \hat{C}, \hat{D} . M=I$ is taken and hence, $N=I-P X$. The controller state-space matrices are then reconstructed 
according to (11a)-(11d) as given below:

$$
\begin{gathered}
A_{c}=\left[\begin{array}{ccccc}
-10.40 & -9.92 & -13.89 & -10.27 & -9.25 \\
-5.73 & -6.89 & -5.02 & -4.09 & -4.20 \\
1.58 & 0.80 & -1.89 & 0.82 & 1.83 \\
9.19 & 2.87 & 3.17 & 1.89 & 6.55 \\
-3.24 & -0.09 & 6.12 & 0.08 & -6.57
\end{array}\right], \\
B_{c}=\left[\begin{array}{ccc}
46.38 & -4.96 & -39.16 \\
-4.96 & -65.26 & -10.10 \\
19.58 & -40.04 & 31.07 \\
20.32 & -8.28 & 17.61 \\
-39.16 & -10.10 & -38.19
\end{array}\right], D_{c}=0_{3 \times 3}, \\
\text { and } C_{c}=\left[\begin{array}{ccccc}
0.27 & 0.36 & 0.31 & 0.36 & 0.28 \\
-0.04 & 0.11 & 0.06 & 0.096 & 0.026 \\
0.39 & 0.23 & -0.24 & 0.20 & 0.38
\end{array}\right]
\end{gathered}
$$

We compute the nominal closed-loop system

$$
\begin{aligned}
& (s+2.35)\left(s^{2}+4.942 s+7.379\right) \\
& \left(s^{2}+13.89 s+49.69\right)\left(s^{2}+6.256 s+13.73\right) \\
& M(s)=\frac{\left(s^{2}+6.423 s+18.4\right)}{(s+2.325)(s+3.274)} \text {, } \\
& \left(s^{2}+4.967 s+7.426\right)\left(s^{2}+6.174 s+13.64\right) \\
& \left(s^{2}+5.95 s+17.95\right)\left(s^{2}+11.17 s+62.71\right)
\end{aligned}
$$

which has been verified to be SNI with $\mathfrak{R}\left[\lambda_{i}\left[A_{c l}\right]\right]<-2$. The Nyquist plot of $M(s)$ shown Fig. 5 confirms that it is indeed SNI. As $M(s)$ is strictly proper and $M(0) \Delta(0)=$ $0.2507 \times 3.6=0.9025<1$, it guarantees that the designed controller $K(s)$ ensures robust stability of the generalized plant $G$ against any stable NI uncertainty $\Delta(s)$ satisfying $\Delta(0) \leq 3.6$ via Theorem 2 .

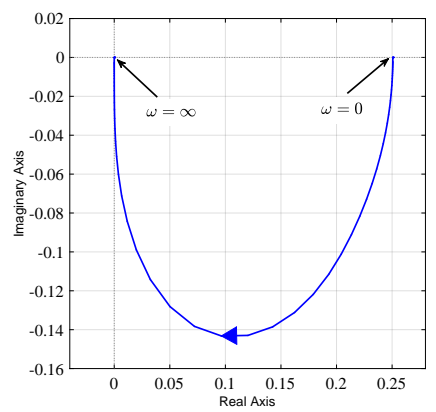

Fig. 5: Nyquist plot of the synthesized $\alpha-$ SNI transfer function $M(s)$ obtained in Example 2.

\section{CONCLUSIONS}

In this paper, we propose an LMI-based $\alpha$ - SNI synthesis technique for stable/unstable LTI systems with stable NI uncertainty. This synthesis technique transforms the nominal closed-loop system into a subset of the SNI class along with the closed-loop poles having $\mathfrak{R}[s] \leq-\alpha$ for a given $\alpha>0$. Robust stability is also guaranteed against any stable strictly proper NI uncertainty satisfying the required DC-gain bound. Moreover, using the proposed technique, other performance measures such as, $\mathscr{H}_{2}$ and $\mathscr{H}_{\infty}$, can be seamlessly added by following the approach presented in [20]. In future, the effectiveness of the controller may be validated in an experimental set-up of a series elastic actuator.

\section{REFERENCES}

[1] A. Lanzon and I. R. Petersen, "Stability robustness of a feedback interconnection of systems with negative imaginary frequency response," IEEE Transactions on Automatic Control, vol. 53, no. 4, pp. 10421046, 2008.

[2] J. Xiong, I. R. Petersen, and A. Lanzon, "A negative imaginary lemma and the stability of interconnections of linear negative imaginary systems," IEEE Transactions on Automatic Control, vol. 55, no. 10, pp. 2342-2347, 2010.

[3] I. R. Petersen and A. Lanzon, "Feedback control of negative imaginary systems," IEEE Control Systems Magazine, vol. 30, no. 5, pp. 54-72, 2010.

[4] C. Cai and G. Hagen, "Stability analysis for a string of couple stable subsystems with negative imaginary frequency response," IEEE Transactions on Automatic Control, vol. 55, no. 8, pp. 1958-1963, 2010.

[5] A. Lanzon, Z. Song, S. Patra, and I. R. Petersen, "A strongly strict negative-imaginary lemma for non-minimal linear systems," Соттиnications in Information and Systems, vol. 11, no. 2, pp. 139-152, 2011.

[6] Z. Song, A. Lanzon, S. Patra, and I. R. Petersen, "A negative imaginary lemma without minimality assumptions and robust statefeedback synthesis for uncertain negative imaginary systems," Systems \& Control Letters, vol. 61, no. 12, pp. 1269-1276, 2012.

[7] Z. Song, A. Lanzon, S. Patra, and I. R. Petersen, "Robust performance analysis for uncertain negative imaginary systems," International Journal of Robust and Nonlinear Control, vol. 22, no. 3, pp. 262-281, 2012.

[8] A. Ferrante and L. Ntogramatzidis, "Some new results in the theory of negative-imaginary systems with symmetric transfer matrix function," Automatica, vol. 49, no. 7, pp. 2138-2144, 2013.

[9] J. Rubi-Masseg, J. M. Rossell, H. R. Karimi, and F. PalaciosQuionero, "Static output-feedback control under information structure constraints," Automatica, vol. 49, no. 1, pp. 313-316, 2013.

[10] M. A. Mabrok, A. G. Kallapur, I. R. Petersen, and A. Lanzon, "Generalizing negative-imaginary systems theory to include free body dynamics: Control of highly resonant structures with free body motion," IEEE Transactions on Automatic Control, vol. 59, no. 10, pp. 2692-2707, 2014.

[11] M. Mabrok, A. G. Kallapur, I. R. Petersen, and A. Lanzon, "A generalized negative imaginary lemma and Riccati-based static state feedback negative imaginary synthesis," Systems \& Control Letters, vol. 77 , pp. 63-68, 2015.

[12] M. Liu and J. Xiong, "On $\alpha$ - and $\mathscr{D}$ - negative-imaginary systems," International Journal of Control, vol. 88, no. 10, pp. 1933-1941, 2015.

[13] J. Xiong, J. Lam, and I. R. Petersen, "Output feedback negative imaginary synthesis under structural constraints," Automatica, vol. 71, pp. 222-228, 2016.

[14] A. Lanzon and H. J. Chen, "Feedback stability of negative imaginary systems," IEEE Transactions on Automatic Control, vol. 62, no. 11, pp. 5620-5633, 2017.

[15] K. Z. Liu, M. Ono, X. Li, and M. Wu, "Robust performance synthesis for systems with positive-real uncertainty and an extension to the negative-imaginary case," Automatica, vol. 82, pp. 194-201, 2017.

[16] G. Salcan-Reyes and A. Lanzon, "On negative imaginary synthesis via solutions to Riccati equations," in Proceedings of European Control Conference, pp. 870-875, Cyprus, June, 2018.

[17] G. Salcan-Reyes, A. Lanzon, and I. R. Petersen, "Controller synthesis to render a closed loop transfer function strongly strictly negative imaginary," in Proceedings of 57th IEEE Conference on Decision and Control, pp. 2988-2993, Miami, USA, Dec 2018.

[18] K. Zhou, J. C. Doyle, and K. Glover, "Robust and Optimal Control," Prentice Hall, Englewood Cliffs, NJ, 1996.

[19] M. Chilali and P. Gahinet, " $\mathscr{H}_{\infty}$ design with pole placement constraints: An LMI approach," IEEE Transactions on Automatic Control, vol. 41 , no. 3, pp. 358-367, 1996.

[20] C. Scherer, P. Gahinet, and M. Chilali, "Multi objective outputfeedback control via LMI optimization," IEEE Transactions on Automatic Control, vol. 42, no. 7, pp. 896-911, 1997.

[21] M. Grant and S. Boyd, CVX: Matlab software for disciplined convex programming, version 2.1, http://cvxr. com/cvx, March 2014. 